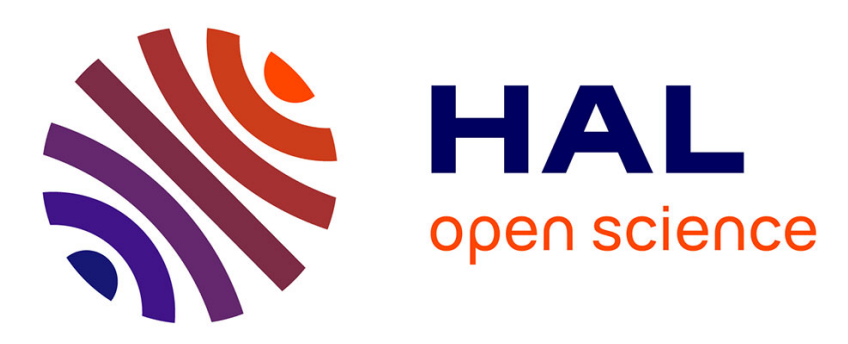

\title{
Permeability Computation on a Representative Volume Element (RVE) of Unidirectional Disordered Fiber Arrays
}

Karim Hitti, Stephanie Feghali, Marc Bernacki

\section{- To cite this version:}

Karim Hitti, Stephanie Feghali, Marc Bernacki. Permeability Computation on a Representative Volume Element (RVE) of Unidirectional Disordered Fiber Arrays. Journal of Computational Mathematics -International Edition-, 2016, 34 (3), pp.233-239. 10.4208/jcm.1511-m2014-0119 . hal-01311282

HAL Id: hal-01311282

https://hal-mines-paristech.archives-ouvertes.fr/hal-01311282

Submitted on 18 Aug 2016

HAL is a multi-disciplinary open access archive for the deposit and dissemination of scientific research documents, whether they are published or not. The documents may come from teaching and research institutions in France or abroad, or from public or private research centers.
L'archive ouverte pluridisciplinaire HAL, est destinée au dépôt et à la diffusion de documents scientifiques de niveau recherche, publiés ou non, émanant des établissements d'enseignement et de recherche français ou étrangers, des laboratoires publics ou privés. 


\title{
PERMEABILITY COMPUTATION ON A REPRESENTATIVE VOLUME ELEMENT (RVE) OF UNIDIRECTIONAL DISORDERED FIBER ARRAYS
}

\author{
Karim Hitti \\ Department of Mathematics, Faculty of Sciences, University of Balamand, Lebanon \\ Notre Dame University-Louaize, Faculty of Natural and Applied Sciences, Department of Mathematics \\ and Statistics, Lebanon \\ Email:khitti@ndu.edu.lb \\ Stephanie Feghali \\ Lebanese University, Faculty of sciences II, Mathematics Department, P.O. Box 90656 Fanar-Matn, \\ Lebanon \\ Email: stephanie.elfeghali@gmail.com \\ Marc Bernacki \\ MINES ParisTech, CEMEF - Centre de Mise en Forme des Matériaux, CNRS UMR 7635, BP 2071 \\ rue Claude Daunesse 06904 Sophia Antipolis cedex, France \\ Email: marc.bernacki@mines-paristech.fr
}

\begin{abstract}
An efficient method to compute the permeability of disordered fibrous arrays is proposed. A stabilized mixed finite element method is used with an immersed domain approach to represent the porous material at its microscopic scale. Therefore, the Stokes equations are solved in the whole domain (including solid part) using a penalization method. The accuracy is controlled by refining the mesh around the fluid-solid interface defined by a level-set function. Using homogenization techniques, the permeability of an RVE is obtained. Furthermore, a new method to generate disordered fibers in function of the porosity, $\phi$, and other microstructural parameters is proposed and a study of the effect of inter-fiber spacing on $\mathcal{K}$, the permeability tensor, is performed. This task was achieved using parallel computation and over 460 simulations were carried out in two-dimensional RVEs consisting of over 555 fibers.
\end{abstract}

Mathematics subject classification: 65N30, 76S05

Key words: Permeability, Fibrous Media, Finite Element Method, Level-set.

\section{Introduction}

Liquid Composite Moulding (LCM) processes are injection processes used for manufacturing large and complex composite materials with fiber reinforcements. Numerical simulation at the macroscopic scale is based on the resolution of Darcy's law [1, 2] to predict flow front progression, filling time and injection pressure and to improve the design of tools and molds.

In the case of ordered fiber arrays, several analytical relations have been established to predict the permeability of fibrous media [3-8]. All of these studies consider simple geometries, such as square or triangular packing of unidirectional arrays of cylinders. And the analytical relations are only a function of $\phi$. By considering some hypothesis, different authors provide 
analytical solutions of the Stokes equations to obtain the permeability. These different laws give a valid solution for different fiber volume fractions. In what concerns numerical analysis, several studies had been done at the microscopic and mesoscopic scales through the finite element or finite volume methods $[4,6,9,10]$. In these studies, fibers or yarns were considered as impermeable solids. Then, only the fluid domain was meshed and no-slip boundary conditions were imposed on solid boundaries. Velocity and pressure fields were computed on the fluid domain and permeability of the volume was then obtained by a homogenization method.

In the case of disordered fiber arrays, which represent real fiber performs, the use of porosity alone cannot define their permeability. Other microstructural parameters should be taken into account. Chen and Papathanasiou [11,12] studied the effect of the mean nearest interfiber spacing, denoted $\bar{\delta}_{1}$, or the degree of disorder, on both the transverse and longitudinal permeabilities. They found that the latter decreases on all porosity levels and the former increases on porosity levels ranging from 0.45 to 0.7 and decreases above these levels when $\bar{\delta}_{1}$ increases. In these studies a Monte-Carlo procedure [13] was used to generate the fiber distributions which are governed by the choice of the porosity and the minimum allowable inter-fiber distance $\delta_{\min }$.

In this paper, we propose an effective method to generate fiber distributions with specific porosities. It is based on advancing front methods [14,15] and dropping and rolling techniques [16]. Also, the inter-fiber distances are chosen either by imposing a $\delta_{\min }$ or generated by a Gaussian distribution law. The influence of the standard deviation of this law, which is proportional to the fiber array's degree of disorder and inversely proportional to Chen and Papathanasiou's $\bar{\delta}_{1}$, on the permeability is studied. Moreover, a new method to compute permeability of a fiber array is proposed where a monolithic approach to solve fluid flow equations is used, followed by a homogenization method to compute permeability on the whole domain. Using a monolithic approach, a unique equation is solved on a mesh containing both fluid and solid domains, and, in our case, using a mixed finite element approximation. The interface is implicitly represented by the zero iso-surface of a level-set function and a penalization method is used to take into account the motion of the solid part [17].

All numerical calculations mentioned in this paper were performed with the CimLib finite element C++ library [18].

\section{Model Equations}

\subsection{Governing equations}

The injected fluid is considered incompressible Newtonian. Due to the fluid's low injection pressure and its high viscosity, inertia and gravity terms can be neglected. Consequently, Stokes equations, describing fluid flow, are written as:

$$
\begin{cases}\eta_{f} \Delta \mathbf{v}-\nabla p & =0 \\ \nabla \cdot \mathbf{v} & =0\end{cases}
$$

with $\mathbf{v}$ the fluid velocity, $p$ the pressure and $\eta_{f}$ the dynamic viscosity.

Darcy's law is traditionally used to model flow motion in porous media at the macroscopic scale. These macroscopic equations are obtained from a volume average of the Navier-Stokes ones $[19,20,21]$, describing the flow motion at the microscopic scale. 
We will use the definitions of the averages given in [21], where the spatial average of a quantity $B$ is defined in the whole volume:

$$
\langle B\rangle=\frac{1}{V} \int_{\Omega} B \mathrm{~d} \Omega
$$

The intrinsic phase average of a quantity $B_{f}$ is defined in the fluid phase:

$$
\left\langle B_{f}\right\rangle^{f}=\frac{1}{V_{f}} \int_{\Omega_{f}} B_{f} \mathrm{~d} \Omega,
$$

with $\Omega_{f}$ the fluid domain.

The porosity $\phi$ and the fiber volume fraction $\phi_{s}$ are defined by the ratio between the volume occupied by the fluid $V_{f}$ and total volume $V$ :

$$
\phi=\frac{V_{f}}{V} \text { and } \phi_{s}=1-\phi .
$$

We suppose that the solid skeleton is static and non-deformable, and that the porous media is saturated. Neglecting effects of viscosity on the flow with respect to the resistance of the skeleton, Darcy's equation is obtained:

$$
\langle v\rangle=-\frac{1}{\eta_{f}} \mathcal{K} \cdot\langle\nabla p\rangle^{f} .
$$

The permeability $\mathcal{K}$ is a tensor for anisotropic porous media, like fibrous media for example. In the case of unidirectional fiber packings, if the $z$ axis of the coordinate system is taken in the same direction as the axis of the fibers, the permeability tensor can be then written as follows:

$$
\mathcal{K}=\left(\begin{array}{cc}
K_{\perp} & 0 \\
0 & K_{\|}
\end{array}\right)
$$

with $K_{\perp}$ a transverse permeability tensor and $K_{\|}$the permeability along the fiber axis. For regular packings (rectangular, triangular or hexagonal) the matrix $K_{\perp}$ is proportional to the unit matrix and then only a scalar $K_{\perp}$ has to be computed. However for disordered arrays, we need to look for a more suitable form:

$$
K_{\perp}=\left(\begin{array}{cc}
K_{x x} & K_{x y} \\
K_{x y} & K_{y y}
\end{array}\right) \text { or } K_{\perp}=\left(\begin{array}{cc}
K_{x x} & 0 \\
0 & K_{y y}
\end{array}\right)
$$

\section{Immersed Domain Method for the Computation of Flow Motion}

First, the Stokes equations (2.1) are solved on the RVE by an immersed domain method [22]. The approach proposed here can rather be seen as an extension of the Stokes problem with two fluids, for which the solid behavior of one phase is imposed by using the viscosity as a penalty factor. All the phases of the multi domain problem are implicitly represented by a level-set function $\alpha$ and the interface between the two phases is defined by its iso-zero [17]. To obtain an accurate description of the interface and small errors concerning the computed flow, efficient anisotropic adaptive meshing tools are used [23]. The viscosity $\eta(\alpha)$ is defined on the whole computational domain using a mixture law [24] (see Figure 3.1). The rigidity of the solid part is taken into account with a high value of the viscosity (namely $\eta_{s}=10^{3} \eta_{f}[25]$ ), this viscosity $\eta_{s}$ acts as a penalty coefficient. Using this approach, a zero velocity boundary condition imposed on the solid's interface is propagated into the entire solid domain. 


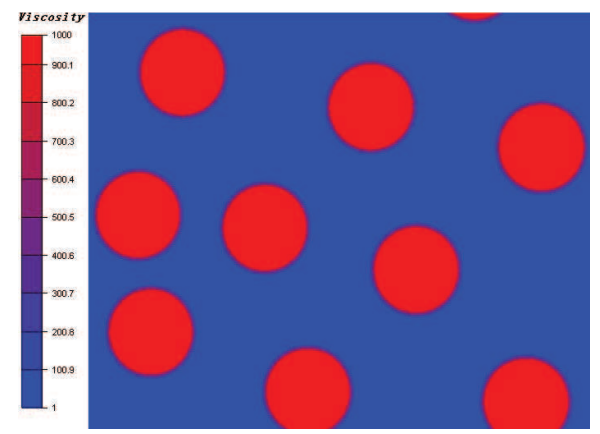

(a)

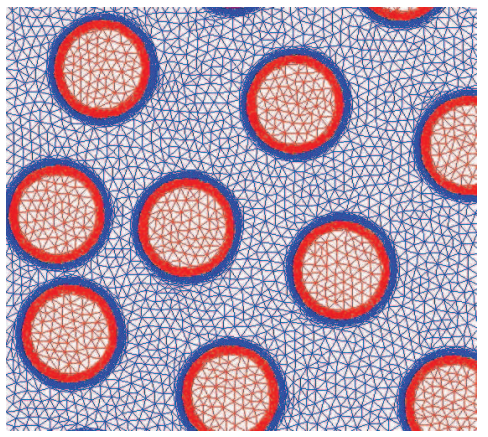

(b)

Fig. 3.1. (a) $\eta(\alpha)$ defined on the whole computational domain using a mixture law and (b) a the anisotropic remeshing at the solid-fluid interface

\subsection{The FE formulation}

First, the function spaces used in this paper are defined. The scalar function space for the pressure $P=\mathcal{L}^{2}(\Omega)$, the function space for the velocity $V=\left(\mathcal{H}^{1}(\Omega)\right)^{d}$ and the weighting function space $V^{0}=\left(\mathcal{H}_{0}^{1}(\Omega)\right)^{d}$, where $d$ is the space dimension. $\mathcal{L}^{2}(\Omega)$ is the Lebesgue space of square assumable functions on $\Omega$ and $\mathcal{H}^{1}(\Omega)$ is the Sobolev space included in $\mathcal{L}^{2}(\Omega)$, are respectively defined by:

$$
\begin{array}{r}
\mathcal{L}^{2}(\Omega)=\left\{q / \int_{\Omega} q^{2} d \Omega<\infty\right\} \\
\mathcal{H}^{1}(\Omega)=\left\{q \in \mathcal{L}^{2}(\Omega) / \nabla q \in \mathcal{L}^{2}(\Omega)\right\} \\
\left.\mathcal{H}_{0}^{1}(\Omega)=\left\{q \in \mathcal{H}^{1}(\Omega) / q=0 \text { on } \partial \Omega\right)\right\}
\end{array}
$$

The FE formulation begins by writing the weak form of the Stokes equations (2.1). The problem consists in finding $(\mathbf{v}, p) \in\left(\mathcal{H}^{1}(\Omega)\right)^{d} \times \mathcal{L}^{2}(\Omega)$ such that:

$$
\left\{\begin{array}{l}
2 \int_{\Omega} \eta(\alpha) \varepsilon(\mathbf{v}): \varepsilon(\mathbf{w}) d \Omega-\int_{\Omega} p \nabla \cdot \mathbf{w} d \Omega=0 \\
\int_{\Omega} q \nabla \cdot \mathbf{v} d \Omega=0
\end{array}\right.
$$

where the trial function $q$ for the pressure is defined in $\mathcal{L}^{2}(\Omega)$ and the trial function $\mathbf{w}$ for the velocity is defined in $\left(\mathcal{H}_{0}^{1}(\Omega)\right)^{d}$.

The domain $\Omega$ is decomposed into $N_{e l}$ triangles $T$ such that they cover the domain and there are either disjoint or share a complete edge. The triangulation will be denoted $\mathcal{T}_{h}$. Using this partition, the above-defined functional spaces are approached by a finite dimensional spaces spanned by continuous piecewise polynomials such that:

$$
\begin{array}{r}
Q_{h}=\left\{q_{h} \in C^{0}(\Omega) / q_{h \mid T} \in P^{1}(T), \forall T \in \mathcal{T}_{h}\right\} \\
V_{h}=\left\{\mathbf{v}_{\mathbf{h}} \in\left(C^{0}(\Omega)\right)^{d} / \mathbf{v}_{\mathbf{h} \mid \mathbf{T}} \in\left(P^{1}(T)\right)^{d}, \forall T \in \mathcal{T}_{h}\right\} \\
V_{h, 0}=\left\{\mathbf{v}_{\mathbf{h}} \in V_{h} / \mathbf{v}_{\mathbf{h} \mid \mathbf{\Gamma}}=0\right\}
\end{array}
$$

where $P^{1}(T)$ is the vector space of first degree polynomials defined on the simplex $T$.

The Galerkin discrete problem consists now in solving the mixed problem by finding the pair $\left(\mathbf{v}_{\mathbf{h}}, p_{h}\right) \in V_{h} \times Q_{h}$ such that $\forall\left(\mathbf{w}_{\mathbf{h}}, q_{h}\right) \in V_{h, 0} \times Q_{h}$ :

$$
\left\{\begin{array}{l}
2 \int_{\Omega} \eta(\alpha) \varepsilon\left(\mathbf{v}_{\mathbf{h}}\right): \varepsilon\left(\mathbf{w}_{\mathbf{h}}\right) d \Omega-\int_{\Omega} p_{h} \nabla \cdot \mathbf{w}_{\mathbf{h}} d \Omega=0 \\
\int_{\Omega} q_{h} \nabla \cdot \mathbf{v}_{\mathbf{h}} d \Omega=0
\end{array}\right.
$$


It is well known that the $P_{1}-P_{1}$ approximation for the discrete Stokes problem (3.4) gives an unstable formulation, since the Brezzi-Babuska condition is not respected [26]. For this reason, we use the MINI-element (see Figure 3.2), in which the functional space $V_{h}$ for the velocity is enriched with the space of the bubble function $V^{\prime}$ :

$$
V^{\prime}=\left\{v^{\prime},\left.v^{\prime}\right|_{T_{i}} \in P^{1}\left(T_{i}\right) \cap \mathcal{H}_{0}^{1}\left(T_{i}\right), \forall T \in \mathcal{T}_{h}, i=1, \ldots, D\right\},
$$

where $D$ is the topological dimension and $T_{i}$ is a decomposition of $T$ in $D$ sub-triangles, that have as a common vertex the barycenter, $G_{T}$, of $T$. In other words, the choice of this bubble function is continuous inside the element, considered as linear on each sub-triangle and vanishes at the boundary of $T$.

The velocity field is now an element of the function space generated by the following direct sum:

$$
\mathcal{V}_{h}=V_{h} \oplus V^{\prime} .
$$

Hence, on each element $T$, the solution takes the form:

$$
\left.\mathbf{v}_{\mathbf{h}}\right|_{T}=\sum_{i}^{D} v_{T}^{i} N_{i}+v_{T}^{\prime} b_{T}
$$

where $N_{i}$ is the interpolation function associated with node $i$. The bubble function $b_{T}$ is defined on each element $T$ as follows: $b_{T}=0$ on $\partial T ; b_{T}\left(G_{T}\right)=1$, Furthermore, $b_{T}$ satisfies the orthogonality condition:

$$
\int_{T} \partial_{x_{k}} N_{i} \partial_{x_{l}} b_{T} d \Omega=0 \forall k, l ; \forall N_{i} \in P^{1}(T) .
$$

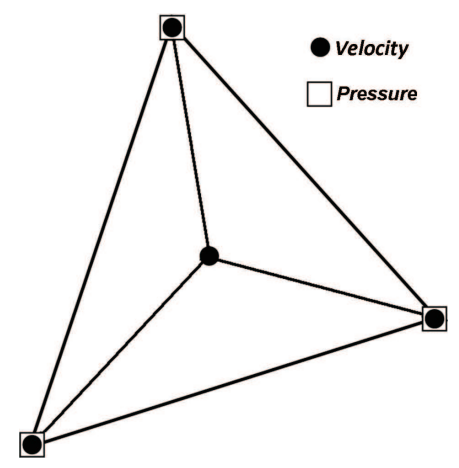

Fig. 3.2. The MINI-element $P_{1}^{+}-P_{1}$

Using the orthogonality properties of the bubble space, the discrete Stokes equation (3.4) is then written:

find $\left(v_{h}, v^{\prime}, p_{h}\right) \in \mathcal{V}_{h} \times Q_{h}$ such that

$$
\left\{\begin{array}{l}
2 \int_{\Omega} \eta(\alpha) \varepsilon\left(\mathbf{v}_{\mathbf{h}}\right): \varepsilon\left(\mathbf{w}_{\mathbf{h}}\right) d \Omega-\int_{\Omega} p_{h} \nabla \cdot \mathbf{w}_{\mathbf{h}} d \Omega=0 \\
2 \int_{\Omega} \eta(\alpha) \varepsilon\left(v^{\prime}\right): \varepsilon\left(w^{\prime}\right) d \Omega-\int_{\Omega} p_{h} \nabla \cdot w^{\prime} d \Omega=0 \\
\int_{\Omega} q_{h} \nabla \cdot\left(\mathbf{v}_{\mathbf{h}}+v^{\prime}\right) d \Omega=0
\end{array}\right.
$$


This system can be put naturally under the following matrix form:

$$
\left(\begin{array}{ccc}
A_{v v} & 0 & A_{v p}^{t} \\
0 & A_{b b} & A_{b p}^{t} \\
A_{v p} & A_{b p} & 0
\end{array}\right)\left(\begin{array}{c}
\mathbf{v}_{\mathbf{h}} \\
v^{\prime} \\
p_{h}
\end{array}\right)=\left(\begin{array}{l}
0 \\
0 \\
0
\end{array}\right)
$$

where:

$$
\begin{gathered}
A_{v v}=2 \int_{\Omega} \eta(\alpha) \varepsilon\left(\mathbf{v}_{\mathbf{h}}\right): \varepsilon\left(\mathbf{w}_{\mathbf{h}}\right) d \Omega, \\
A_{b b}=2 \int_{\Omega} \eta(\alpha) \varepsilon\left(v^{\prime}\right): \varepsilon\left(w^{\prime}\right) d \Omega, \\
A_{v p}=\int_{\Omega} p_{h} \nabla \cdot \mathbf{w}_{\mathbf{h}} d \Omega, \\
A_{b p}=\int_{\Omega} p_{h} \nabla \cdot v^{\prime} d \Omega .
\end{gathered}
$$

Finally, the static condensation process [27] consisting in solving the second line for the bubble function $v^{\prime}$ which by inserting into the third line of (3.10) results the final matrix system to solve which is the condensed matrix scheme for large-scale unknowns $\mathbf{v}_{\mathbf{h}}$ and $p_{h}$ reading:

$$
\left(\begin{array}{cc}
A_{v v} & A_{v p}^{t} \\
A_{v p} & C
\end{array}\right)\left(\begin{array}{c}
\mathbf{v}_{\mathbf{h}} \\
p_{h}
\end{array}\right)=\left(\begin{array}{l}
0 \\
0
\end{array}\right)
$$

with $C=-A_{b p} A_{b b}^{-1} A_{b p}^{t}$.

\section{The Representative Volume Element (RVE)}

In order to get suitable averaged quantities describing the macroscopic behavior of the material, it is necessary to perform computations on an RVE. The RVE is a two dimensional rectangle of width, $L$ and height $H$ on which three different boundary conditions are applied:

1. the pressure gradient is in the horizontal direction $x, v_{y}$ is null on the external boundary and

$$
\partial_{y} v_{x}(x, 0)=\partial_{y} v_{x}(x, H)=0 ; v_{z}(x, y)=0, \partial_{z} p(x, y)=0 ; p(0, y)=P, p(L, y)=0
$$

2. the pressure gradient is in the vertical direction $y, v_{x}$ is null on the external boundary and

$$
\partial_{y} v_{y}(0, y)=\partial_{y} v_{y}(L, y)=0 ; v_{z}(x, y)=0, \partial_{z} p(x, y)=0 ; p(x, 0)=P, p(x, H)=0 .
$$

3. the pressure gradient is in the longitudinal direction $z, v_{x}$ and $v_{y}$ are null,

$$
\partial_{x} v_{z}(0, y)=\partial_{x} v_{z}(L, y)=0 ; \partial_{y} v_{z}(x, 0)=\partial_{y} v_{z}(x, H)=0 ; \partial_{z} p(x, y)=P / L .
$$




\subsection{Disordered packing}

In order to generate disordered fiber packing with pre-chosen porosity, radius and inter-fiber spacing, a sphere packing method called the "Inwards Packing Method (IPM)" [14,15], coupled with a "dropping and rolling" algorithm $[16,28]$ and a disc shrinking technique, were chosen to generate the initial medium. At first, tangent discs are generated with radii bigger than the wanted radius and equal to $R+\delta_{i} / 2$ where $R$ is the wanted radius and $\delta_{i}$ is the $i^{\text {th }}$ inter-fiber spacing. And then, the radii are decreased by removing the $\delta_{i} / 2$ leaving for two fibers $f_{i}$ and $f_{j}$, which were in contact after the IPM and "dropping and rolling" algorithms, with an inter-fiber spacing of $\frac{\delta_{i}+\delta_{j}}{2}$. Furthermore, the choice of $\delta_{i}$ has a great influence on the porosity and on the degree of disorder of the fiber arrays. Hence, we propose the use of a Gaussian distribution law for the choice of the inter-fiber spacings. The reason for choosing a Gaussian distribution law comes from the fact that, contrary to other classical probability distributions, the sum of independent Gaussian distributions $N\left(\mu_{i}, \sigma_{i}^{2}\right)$ is the Gaussian distribution $N\left(\sum_{i} \mu_{i}, \sum_{i} \sigma_{i}^{2}\right)$. So when the inter-fiber distance is wanted according to $N\left(\delta, \sigma^{2}\right)$, it is actually generated following $N\left(\delta / 2, \sigma^{2} / 2\right)$ and added to the wanted radius then when subtracted, the inter-fiber distance between two fibers will follow $N\left(\delta, \sigma^{2}\right)$. Furthermore, a simple, yet efficient, choice of $\delta_{\text {mean }}$, which is the mean of the Gaussian distribution law, leads to obtaining the wanted porosity. This choice is influenced by the fact that the optimal density, $\rho_{\text {opt }}$, of equally spaced discs (i.e. the hexagonally packed) is given by (see Figure 4.1):

$$
\rho_{\text {opt }}=\frac{2 \pi R^{2}}{\sqrt{3}(2 R+\delta)^{2}} .
$$

But since the IPM does not reach the optimal density, this equation is multiplied by $\rho(R)$ which is the disc density obtained by the IPM using the radius $R$.

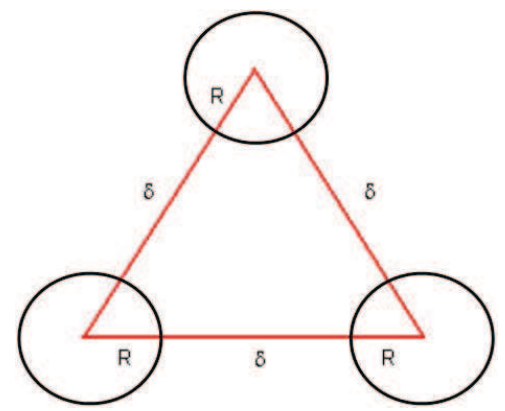

Fig. 4.1. Three equally spaced discs of radius $\mathrm{R}$ and their equilateral disposition

Finally, the wanted porosity, equal to $1-\rho_{\text {opt }} . \rho(R)$, yields that:

$$
\delta_{\text {mean }}=R\left(\sqrt{\frac{2 \pi \rho(R)}{(1-\phi) \sqrt{3}}}-2\right) .
$$

The standard deviation of the Gaussian distribution is written $\sigma=\frac{\delta_{\text {mean }}-\delta_{\min }}{3}$, where $\delta_{\text {min }}$ is the minimal allowable inter-fiber spacing. Typical fiber distributions are shown in Figure 4.2 for different values of $\phi$ and $\sigma$.

It is noticed that $\sigma$ has a greater effect on the fiber distribution when the porosity is large and that it is proportional to the fiber array's degree of disorder. Moreover, by varying $\sigma$ a 


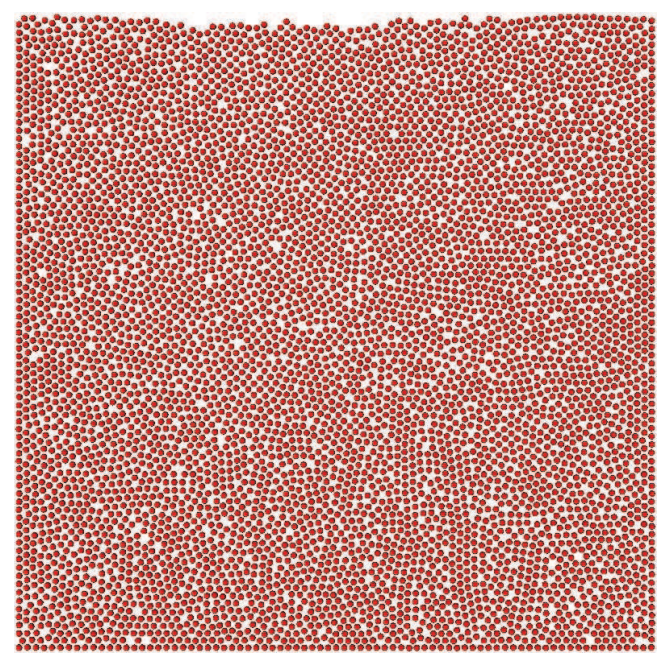

(a)

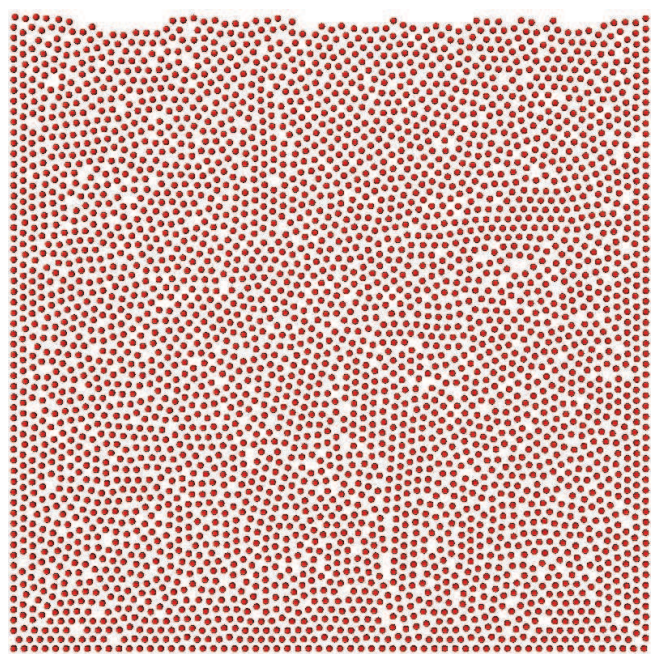

(c)

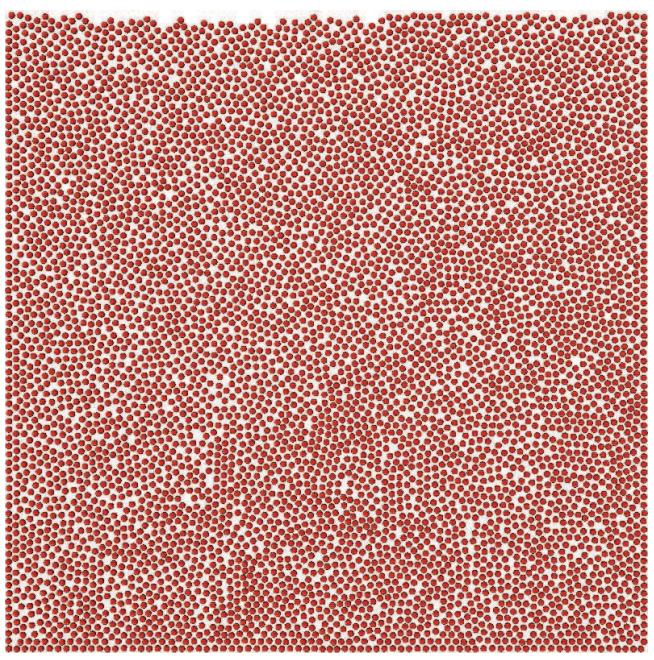

(b)

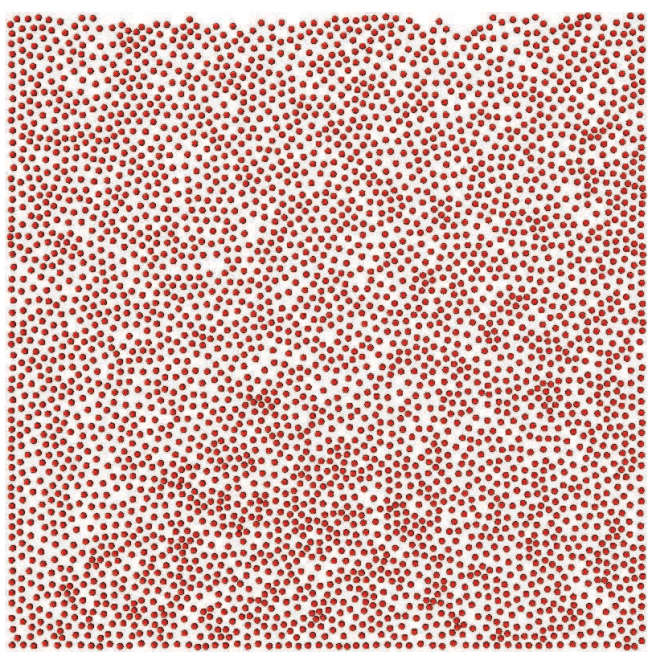

(d)

Fig. 4.2. Fiber distributions generated by our method in a $10 \times 10$ square: (a) 6130 fibers with $\phi=0.51$ and $\sigma=0.06 R$,(b) 6111 fibers with $\phi=0.51$ and $\sigma=0.12 R$, (c) 3678 fibers with $\phi=0.7$ and $\sigma=0.03 R$ and (d) 3634 fibers with $\phi=0.7$ and $\sigma=0.4 R$

spectrum of fiber distributions can be generated at the same $\phi$ which means that this standard deviation is linked to the microstructure heterogeneity and gives a good insight into the fiber microstructure.

\subsubsection{Algorithm of fiber packing generation}

1. The wanted fiber radius $R$ and inter-fiber spacing are chosen. And $\rho_{\text {opt }}$ is computed by $(4.4)$.

2. The IPM is performed using the radius $R$ to get $\rho(R)$.

3. $\delta_{\text {mean }}$ and $\sigma$ of the Gaussian distribution are computed so that the $\delta_{i}$ will follow $N\left(\delta_{\text {mean }} ; \sigma\right)$. 
4. The IPM coupled with dropping and rolling is used to generate discs, representing the fibers, with radii $R+\delta_{i} / 2$.

5. The discs are shrinked by removing $\delta_{i} / 2$ and thus obtaining the wanted fiber packing.

\subsubsection{Ripley's $K_{r}$}

As one of the objectives of this study is to compute the permeability of different random fiber arrangements as a function of other parameters beyond porosity, the randomness of the microstructure needs to be properly quantified. The Ripley's $K_{r}$ function $[29,30]$ can be used to differentiate between regular, Completely Spatially Random (CSR), and clustered point patterns. This function is defined as:

$$
K_{r}(r)=\frac{A}{N^{2}} \sum_{k=1}^{N} \frac{I_{k}(r)}{w_{k}(r)} .
$$

where $I_{k}(r)$ is the number of points found within a distance $r$ of the point $k, N$ is the total number of points in the area of interest $A$, and $w_{k}(r)$ is a correction factor taking account of the fact that it is possible that only a part of the observation area $\pi r^{2}$ falls within the area of interest $A$. This correction factor is computed as the proportion of the perimeter of the circle centered in $k$ with radius $r$ which is inside the area of interest [31]. $K_{r}(r)$ describes characteristics of point patterns at many length scales. The $K_{r}$-function of a Poisson distribution is $\pi r^{2}$ and draws a dividing line between a regular and a clustered pattern. Estimates of $K_{r}(r)$ are expected to be smaller than $\pi r^{2}$ if the points form a regular pattern, and to be larger than $\pi r^{2}$ in the presence of clustering [32]. Furthermore, the extent of the deviation of $K_{r}(r)$ from $\pi r^{2}$ and the length scale at which such deviations occur give some additional insight into a microstructure. The $L$-plot, a linearized plot of $K_{r}(r)$ defined as: $L(r)=\sqrt{K_{r}(r) / \pi}$, is frequently used to show these deviations and the length scales at which they occur. The $L$-plot of a Poisson distribution is simply a straight line of 45 -degree slope through the origin. Figure 4.3 shows the resulting $L(r)$-function applied to the fiber distributions in Figure 4.2. It is evident that at large distances all fiber distributions approach the CSR pattern. This concludes that our method can generate random distributions usable in this type of studies.

\section{Algorithm of Ripley's $K_{r}(r)$}

1. for $r=0$ to $20 *$ fiber's radius

$K_{r}(r)=0$

for $i=0$ to Number of fibers $\left(N_{f}\right)$

a) $I(r)=0$

b) Compute the distance, $d$, between fiber $_{i}$ and fiber $_{j}(i \neq j)$

if $d \leq r$

$I(r)=I(r)+1$

end if

c) Compute the edge correction factor $w(r) ; 28$ cases exist (see [31])

end for $i$

$K_{r}(r)=\frac{\text { Area of } R V E}{N_{f}^{2}} \sum_{i=1}^{N_{f}} \frac{I(r)}{w(r)}$ 
end for $r$

2. Compute $L(r)$

3. Compare with the L-plot of the Poisson distribution

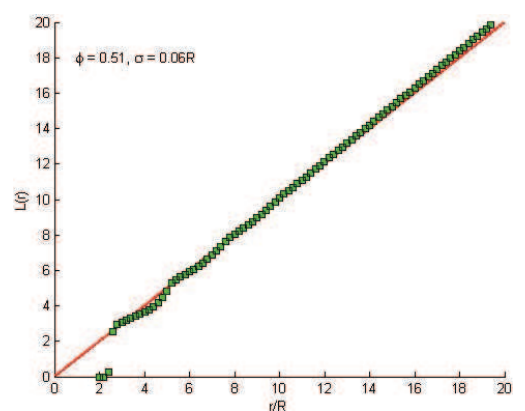

(a)

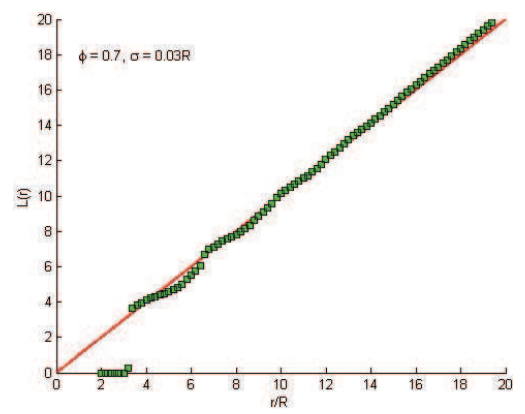

(c)

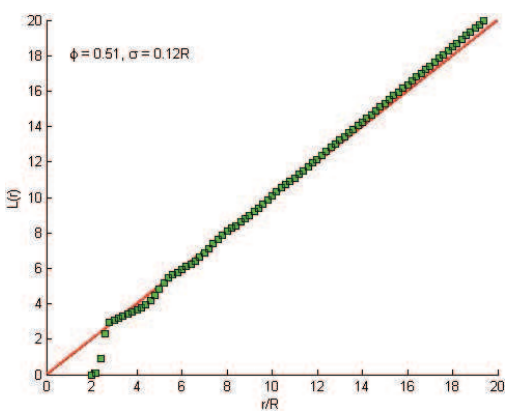

(b)

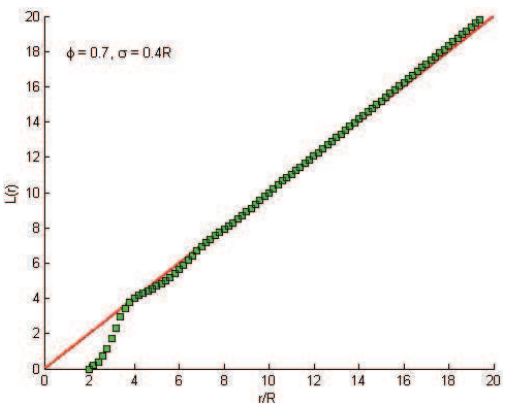

(d)

Fig. 4.3. The $L(r)$ functions of the fiber distributions described in Figure 4.2

\section{Permeability Computation}

\subsection{Computation of the transverse permeability tensor}

We have chosen to use the least squares method to compute the transverse permeability. The computations are performed with the first two sets of boundary conditions for 2D Stokes equations (2.1).

First assuming that the permeability tensor $K_{\perp}$ has the form

$$
K_{\perp}=\left(\begin{array}{ll}
K_{x x} & K_{x y} \\
K_{x y} & K_{y y}
\end{array}\right)
$$

and second, that 2 computations were performed which gives $\left\langle\mathbf{v}_{i}\right\rangle$ and $\left\langle\nabla p_{i}\right\rangle^{f}$ with $i=1,2$.

The least square method consists in minimizing the function $J$

$$
\begin{aligned}
J\left(K_{x x}, K_{y y}, K_{x y}\right)= & \sum_{i=1}^{2}\left(K_{x x}\left\langle\partial_{x} p_{i}\right\rangle^{f}+K_{x y}\left\langle\partial_{y} p_{i}\right\rangle^{f}+\eta_{f}\left\langle v_{x, i}\right\rangle\right)^{2} \\
& +\left(K_{x y}\left\langle\partial_{x} p_{i}\right\rangle^{f}+K_{y y}\left\langle\partial_{y} p_{i}\right\rangle^{f}+\eta_{f}\left\langle v_{y, i}\right\rangle\right)^{2} .
\end{aligned}
$$


The minimum of Eq. (5.2) is obtained when the partial derivatives of the function $J$ with respect to the permeability components are zero i.e.

$$
\begin{gathered}
\frac{\partial J}{\partial K_{x x}}=0, \\
\frac{\partial J}{\partial K_{y y}}=0, \\
\frac{\partial J}{\partial K_{x y}}=0 .
\end{gathered}
$$

This leads to the following system of equations:

$$
\begin{aligned}
A K_{x x}+C K_{x y} & =E, \\
B K_{y y}+C K_{x y} & =F, \\
C K_{x x}+C K_{y y}+(A+B) K_{x y} & =G,
\end{aligned}
$$

with

$$
\begin{aligned}
A & =\sum_{i=1}^{2}\left(\left\langle\partial_{x} p_{i}\right\rangle^{f}\right)^{2}, \\
B & =\sum_{i=1}^{2}\left(\left\langle\partial_{y} p_{i}\right\rangle^{f}\right)^{2}, \\
C & =\sum_{i=1}^{2}\left\langle\partial_{x} p_{i}\right\rangle^{f}\left\langle\partial_{y} p_{i}\right\rangle^{f}, \\
E & =-\eta_{f} \sum_{i=1}^{2}\left\langle\partial_{x} p_{i}\right\rangle^{f}\left\langle v_{x, i}\right\rangle, \\
F & =-\eta_{f} \sum_{i=1}^{2}\left\langle\partial_{y} p_{i}\right\rangle^{f}\left\langle v_{y, i}\right\rangle, \\
G & =-\eta_{f} \sum_{i=1}^{2}\left(\left\langle\partial_{y} p_{i}\right\rangle^{f}\left\langle v_{x, i}\right\rangle+\left\langle\partial_{x} p_{i}\right\rangle^{f}\left\langle v_{y, i}\right\rangle\right),
\end{aligned}
$$

which gives

$$
\begin{aligned}
K_{x x} & =\frac{-B C G+C^{2} F-C^{2} E+E A B+E B^{2}}{d}, \\
K_{y y} & =\frac{A^{2} F-C A G+C^{2} E-C^{2} F+A B F}{d} \\
K_{x y} & =\frac{B A G-B C E-F A C}{d}
\end{aligned}
$$

where $d=(A+B)\left(A B-C^{2}\right)$.

If $K_{x y}$ is assumed to be zero, then,

$$
\begin{aligned}
K_{x x} & =\frac{E}{A}, \\
K_{y y} & =\frac{F}{B},
\end{aligned}
$$


If $K_{x y}=0$ and $K_{x x}=K_{y y}$, a scalar transverse permeability $K$ can be computed as

$$
\begin{aligned}
K & =\frac{E+F}{A+B}=-\eta_{f} \frac{\sum_{i=1}^{2}\left\langle\partial_{x} p_{i}\right\rangle^{f}\left\langle v_{x, i}\right\rangle+\left\langle\partial_{y} p_{i}\right\rangle^{f}\left\langle v_{y, i}\right\rangle}{\sum_{i=1}^{2}\left(\left\langle\partial_{x} p_{i}\right\rangle^{f}\right)^{2}+\left(\left\langle\partial_{y} p_{i}\right\rangle^{f}\right)^{2}} \\
& =-\eta_{f} \frac{\sum_{i=1}^{2}\left\langle\mathbf{v}_{i}\right\rangle \cdot\left\langle\nabla p_{i}\right\rangle^{f}}{\sum_{i=1}^{2}\left\langle\nabla p_{i}\right\rangle^{f} \cdot\left\langle\nabla p_{i}\right\rangle^{f}} .
\end{aligned}
$$

\subsection{Computation of longitudinal permeability}

The longitudinal permeability is obtained by computing the scalar $v_{z}$ solution of the reduced Stokes equation

$$
\eta_{f}\left(\frac{\partial^{2} v_{z}}{\partial y^{2}}+\frac{\partial^{2} v_{z}}{\partial x^{2}}\right)=\frac{\partial p}{\partial z} \text { on } \Omega_{f}
$$

and

$$
K_{\|}=-\eta_{f} \frac{\left\langle v_{z}\right\rangle}{\partial_{z} p}
$$

\section{Numerical Results}

\subsection{The RVE's size}

The randomness of the generated fiber distributions leads to a scatter in the computed permeability. Hence, an average permeability should be calculated along with a data variation in order to be statistically representative. In this work, 20 random microstructures were generated for each class of fiber distributions characterized by the same $\phi$ and $\sigma$. The average permeability and its standard deviation, $\sigma_{p}$, are given by:

$$
\begin{gathered}
\langle K\rangle=\frac{1}{20} \sum_{i=1}^{20} K_{i}, \\
\sigma_{p}=\frac{1}{19} \sqrt{\sum_{i=1}^{20}\left(K_{i}-\langle K\rangle\right)^{2} .}
\end{gathered}
$$

Moreover, the permeability of fiber distributions is not only a function of microstructural parameters but also a function of the RVE's size which is the same as the number of fibers $N_{f}$ in the domain. An extremely large computational domain is surely representative of the fiber array but this will cause a drastic increase in the computational cost which makes this type of studies quasi-impossible. In order to determine the appropriate size of the RVE a study of the size effect on $\langle K\rangle,\left\langle K_{\|}\right\rangle$and $\phi$ was performed and computations were carried out on elementary volumes of different size extracted from microstructures generated with $\sigma=0.06 R$ and $\phi=0.5$ as illustrated in Figure 6.1.

The results are shown in Figure 6.2 where the permeability values are adimensionalised.

Fluctuations are observed in the values of $\langle K\rangle / R^{2},\left\langle K_{\|}\right\rangle / R^{2}$ and $\langle\phi\rangle$ when a relatively small number of fibers is considered then the values reach a plateau after $N_{f}>385$. Also, after $N_{f}>555$ the associated standard deviations are reduced. As a conclusion, an RVE's size of $N_{f}>555$ is used in the remainder of this paper. 


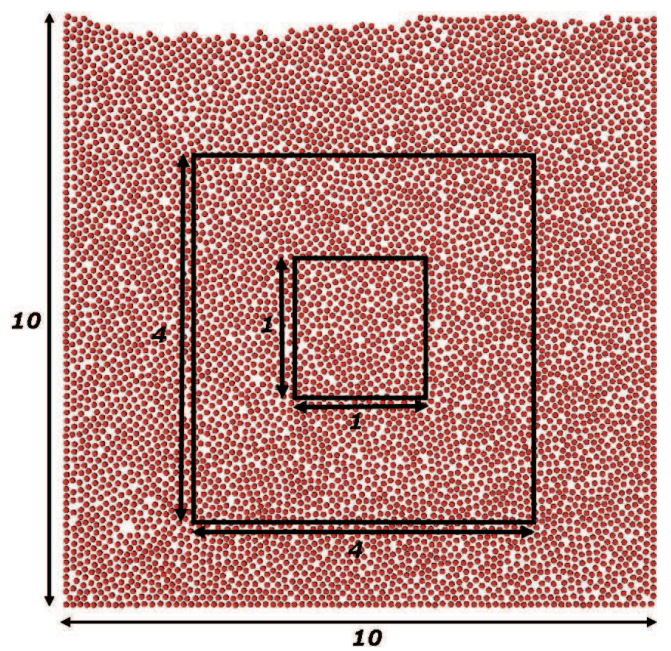

Fig. 6.1. Elementary volumes extracted from a $10 \times 10$ microstructure in order to determine the size of the RVE

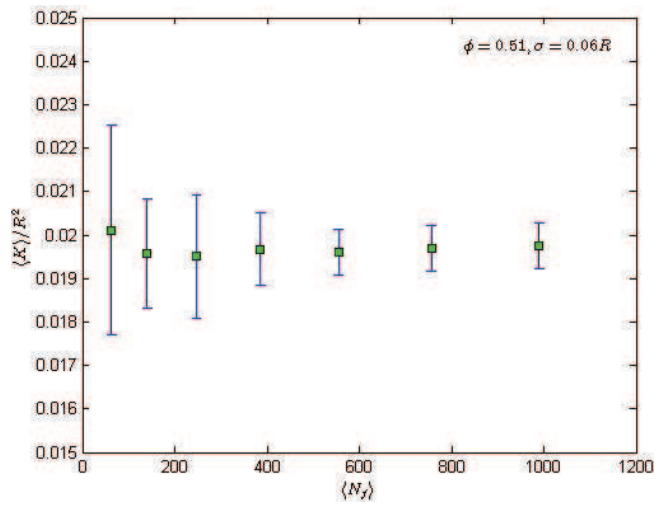

(a)

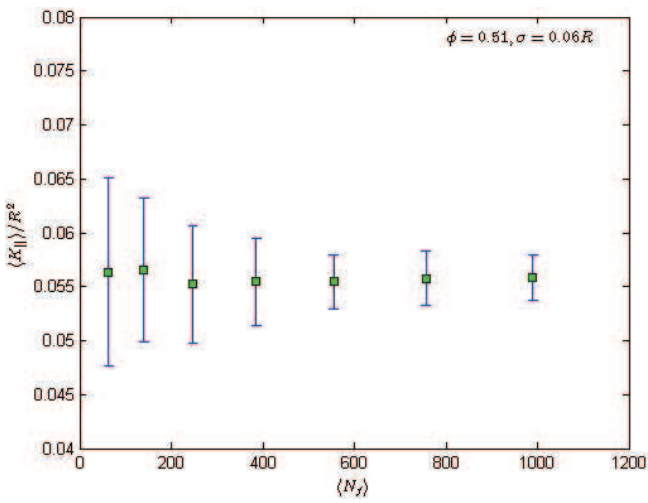

(b)

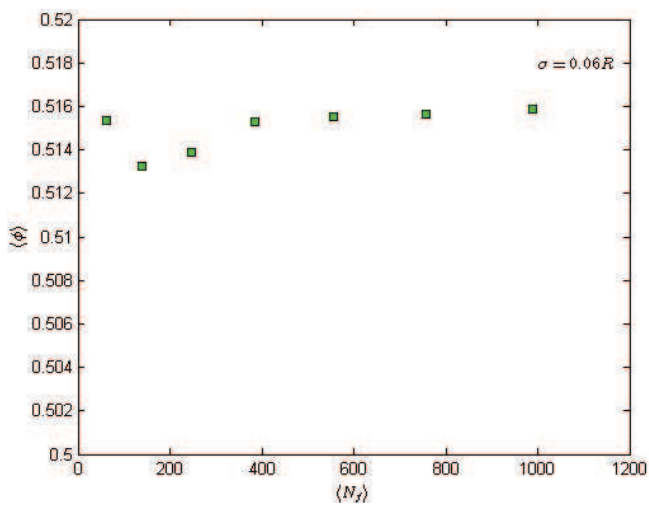

(c)

Fig. 6.2. The size effect on $\langle K\rangle / R^{2}$. The fiber distributions were generated with $\sigma=0.06 R$ and $\phi=0.51$. The error bars represent the standard deviations $\pm \sigma_{p}$ 


\subsection{Isotropic properties}

We computed the values of the components of the transverse permeability tensor $K_{\perp}, K_{x x}$, $K_{y y}$ and $K_{x y}$, in order to see if the scalar transverse permeability $K$ can be computed. In Figure 6.3 , the averaged components are computed at different porosity levels and plotted against $\sigma / R$. The values at different porosities are illustrated in different figures for sake of clarity.

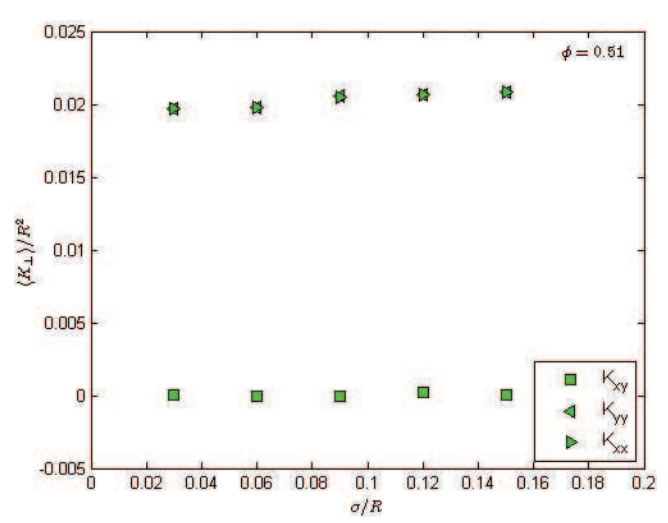

(a)

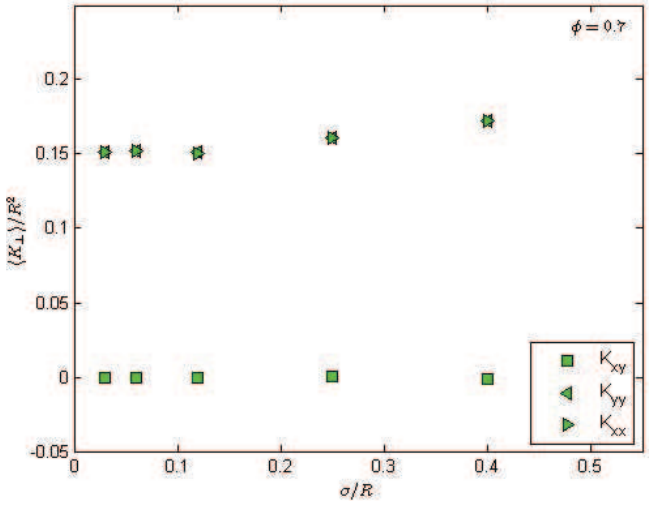

(b)

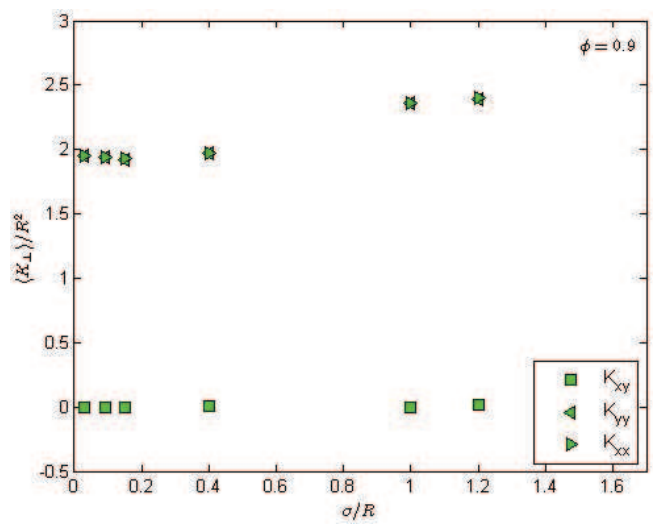

(c)

Fig. 6.3. The averaged permeability components at different values of $\sigma$ : (a) $\phi=0.51$, (b) $\phi=0.7$ and (c) $\phi=0.9$

Figure 6.3 shows that $K_{x x}$ is equal to $K_{y y}$ and $K_{x y}$ is negligible on all porosity levels and at all values of $\sigma$. This means that the transverse permeability is independent of the flow direction and that the fiber arrays are isotropic. In this case, a scalar transverse permeability is computed by Eq.(5.5).

\subsection{The effect of $\sigma$ on $\mathcal{K}$}

Figure 6.4 shows two representative flow paths computed on RVEs at $\phi=0.7$ and Figure 6.5 illustrates the velocity computed for the boundary conditions of type 3 (Eq.(4.3)) on the same previous RVEs. The difference between the two microstructures lies in the choice of $\sigma$ $(\sigma=0.03 R$ in Figures 6.4(a) and 6.5(a) and $\sigma=0.4 R$ in Figures 6.4(b) and 6.5(b)).

We notice from these two figures that the microstructure has a great effect on the flow 


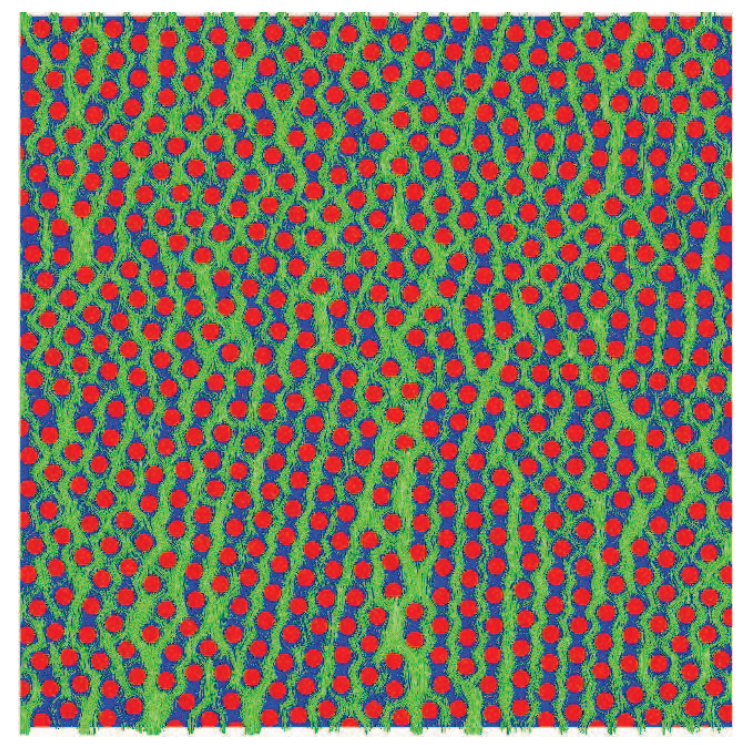

(a)

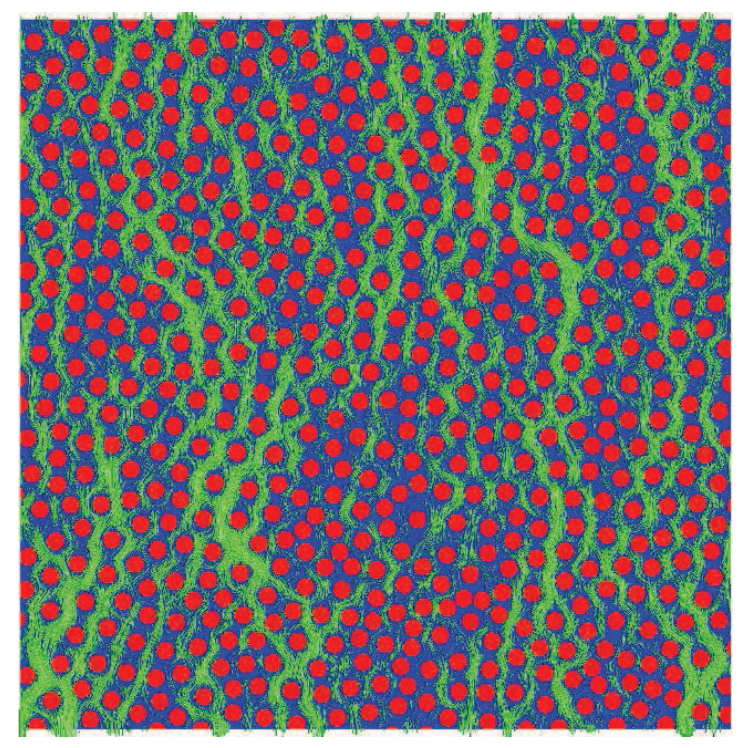

(b)

Fig. 6.4. Flow paths for boundary conditions 2 across a unidirectional disordered fiber array: (a) $\phi=0.7, \sigma=0.03 R$ and (b) $\phi=0.7, \sigma=0.4 R$

distribution in the interstitial space. In what concerns transverse flows (boundary conditions 2 and Figure 6.4), a larger degree of local heterogeneity caused by an increase of $\sigma$ results in a wider range of flow paths. In this case, a few major flow paths exist while in the case of $\sigma=0.03 R$ a large number of small paths exist giving a uniform flow through the fiber array. In what concerns longitudinal flows (boundary conditions 3 and Figure 6.5), a larger degree of local heterogeneity results in the formation of pockets of high-speed fluid against a more uniform speed for small values of $\sigma$. From these Figures, it is evident that fibers at the same porosity can exhibit drastically different patterns of fluid flows. It follows that the use of porosity alone 


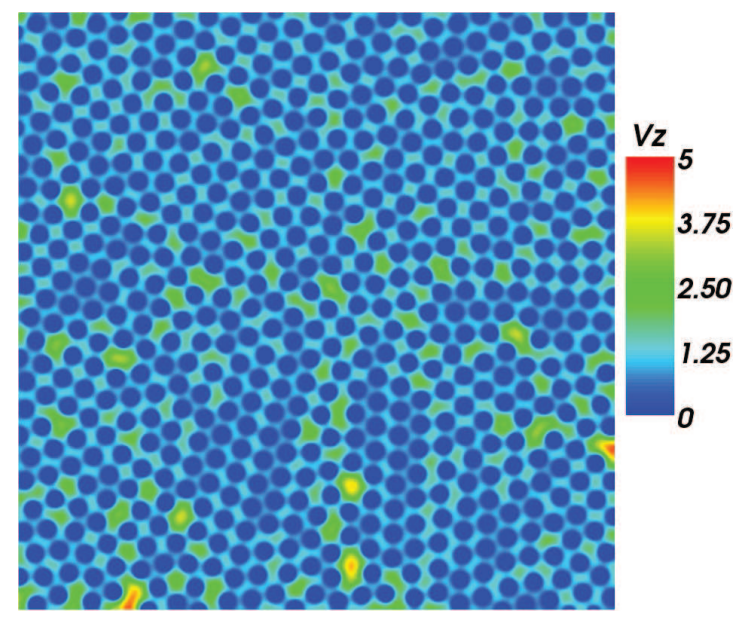

(a)

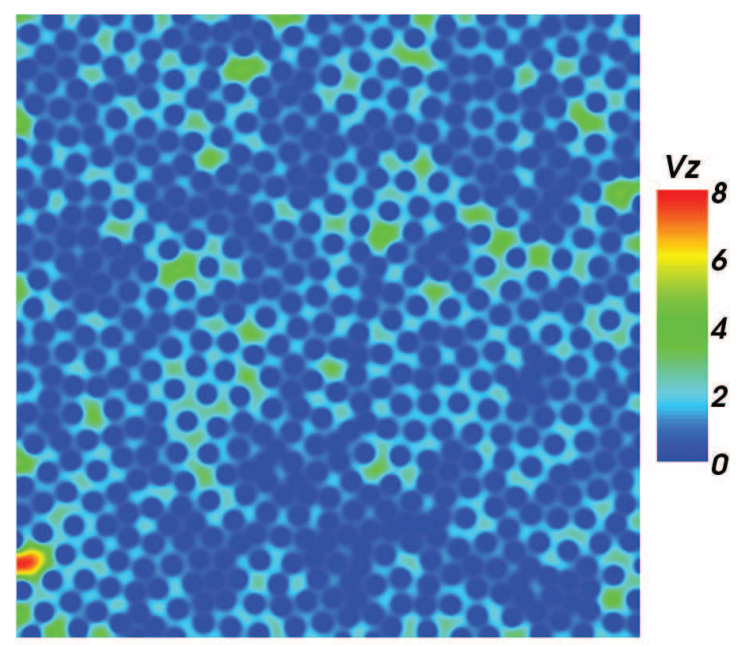

(b)

Fig. 6.5. Contours of fluid velocity for longitudinal flows across a unidirectional disordered fiber array: (a) $\phi=0.7, \sigma=0.03 R$ and (b) $\phi=0.7, \sigma=0.4 R$

cannot define properly their permeability.

In Figure 6.6 the numerically computed $\langle K\rangle$ and $\left\langle K_{\|}\right\rangle$at each porosity level are plotted against $\sigma / R$.

The effect of $\sigma / R$ on $\langle K\rangle / R^{2}$ and $\left\langle K_{\|}\right\rangle / R^{2}$ is evident. An increasing $\sigma$ or also moving from a uniform array to an array showing higher degrees of disorder results in an increase of both permeability values, the transverse and the longitudinal. The results of $\left\langle K_{\|}\right\rangle$are in agreement with those of Chen and Papathanasiou [11] who also found that an increase of the microstructure's heterogeneity enhances $\left\langle K_{\|}\right\rangle$. This is due to the formation of large flow paths. On the other hand, our results concerning $\langle K\rangle$ are opposite to those of Chen and Papathanasiou [12] except for $\phi>0.7$. They explain that the decrease in $\langle K\rangle$ in the range $0.45<\phi<0.7$ comes from the presence of narrow gaps which reduce the permeability and that the increase of $\langle K\rangle$ in the range $\phi>0.7$ comes from the formation of flow paths whose size is comparable to the size of fiber aggregates. In our case, we explain this increase by the simple fact that large gaps 


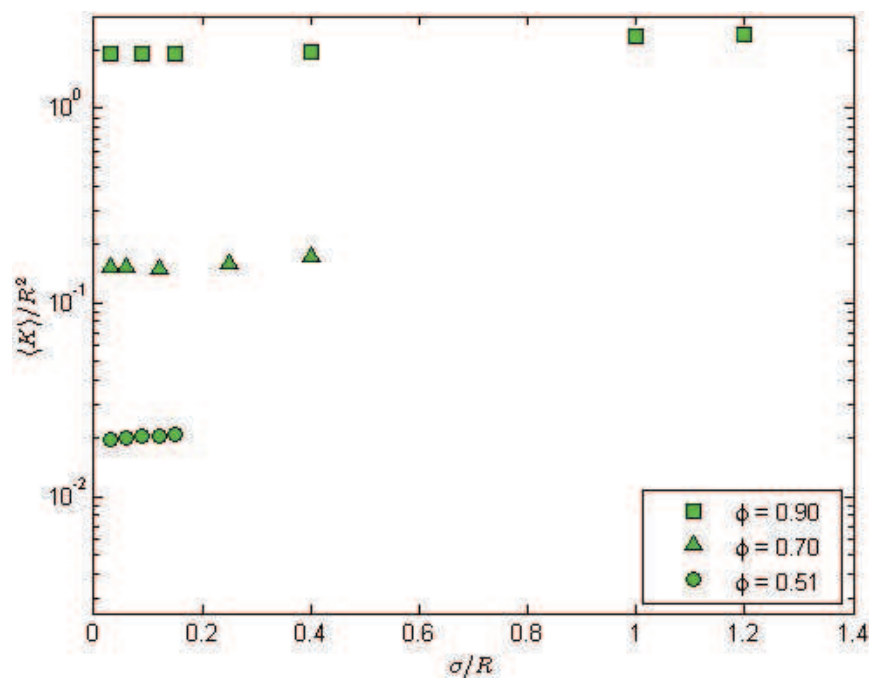

(a)

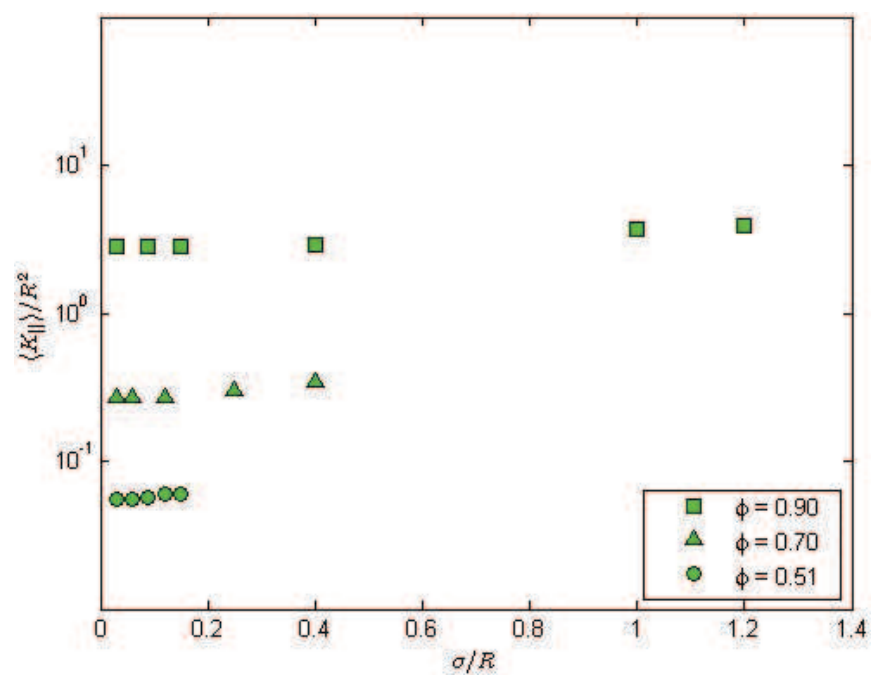

(b)

Fig. 6.6. The effect of averaged standard deviation of the inter-fiber spacing on the normalized averaged transverse (a) and longitudinal (b) permeability

enhance $\langle K\rangle$ as they enhance $\left\langle K_{\|}\right\rangle$.

\section{Conclusion}

A method to generate unidirectional disordered fiber arrays, with a specific predefined porosity and inter-fiber spacing, in an FE context was introduced. This method is based on an advancing front method to generate the fibers' centers, on level-set functions to immerse the fibers in the FE mesh and on anisotropic mesh adaptation to properly describe the fibers' interfaces. Furthermore, permeability computations of the generated unidirectional disordered fiber arrays were performed. The flow through the fibers, which were considered as rigid discs, is governed by the Stokes equations that, when averaged, lead to Darcy's law. Hence, the permeability of a 
fiber array can be computed. The RVE's size, or number of fibers, was determined by studying its influence on the transverse and longitudinal permeabilities. Afterwards, we have shown that the fiber arrays are isotropic and that the transverse permeability is independent of the flow direction. Furthermore, the influence of the degree of disorder of fiber arrays on transverse and longitudinal permeabilities was studied and the results have shown that both permeabilities increase on all porosity levels as the disorder increases.

\section{References}

[1] J. Bear, Dynamics of fluids in porous media, Elsevier, 1972.

[2] H. Darcy, Les fontaines publiques de la ville de Dijon, Dalmont, Victor, 1856.

[3] P.C. Carman, L'écoulement des gaz à travers les milieux poreux, Institut National des Sciences et Techniques Nucléaires, 1961.

[4] B.R. Gebart, Permeability of unidirectional reinforcements for RTM, J. Compos. Mater., 26:8 (1992), 1100-1133.

[5] J. Happel, Viscous flow relative to arrays of cylinders, AIChE. J., 5 (1959), 174-177.

[6] C. Hoareau, Injection sur renfort : Etude du remplissage de moule et détermination théorique de la perméabilité des tissus, PhD thesis, ENSMP, 1994.

[7] J.B. Keller, Viscous flow through a grating or lattice of cylinders, J. Fluid. Mech., 18 (1964), 94-96.

[8] A. Tamayol and M. Bahrami, Analytical determination of viscous permeability of fibrous porous media, Int. J. Heat. Mass. Trans., 52 (2009), 2407-2414.

[9] M. Nordlund and T.S. Lundstrm, Numerical study of the local permeability of noncrimp fabrics, J. Compos. Mater., 39:10 (2005), 929-947.

[10] Y.S. Song and J.R. Youn, Asymptotic expansion homogenization of permeability tensor for plain woven fabrics, Compos. Part A., 37 (2006), 2080-2087.

[11] X. Chen and T.D. Papathanasiou, Micro-scale modeling of axial flow through unidirectional disordered fiber arrays, Compos. Sci. Technol., 67:7-8 (2007), 1286-1293.

[12] X. Chen and T.D. Papathanasiou, The transverse permeability of disordered fiber arrays: a statistical correlation in terms of the mean nearest interfiber spacing, Transport Porous Med., $\mathbf{7 1}$ (2008), 233-251.

[13] S. Torquato, Random Heterogeneous Materials: Microstructure and Macroscopic Properties, Springer- Verlag, 2002.

[14] K. Bagi, An algorithm to generate random dense arrangements for discrete element simulations of granular assemblies, Granul. Matter, 7 (2005), 31-43.

[15] A. Benabbou, H. Borouchaki, P. Laug, and J. Lu, Numerical modeling of nanostructured materials, Finite Elem. Anal. Des., 46:1-2 (2010), 165-180.

[16] K. Hitti and M. Bernacki, Optimized Dropping and Rolling (ODR) method for packing of polydisperse spheres, App. Math. Mod., 37 (2013), 5715-5722.

[17] T. Coupez, H. Digonnet, E. Hachem, P. Laure, L Silva and R. Valette. Multidomain finite element computations: Application to multiphasic problems, Arbitrary Lagrangian-Eulerian and FluidStructure Interaction, Numerical Simulation, Wiley (2010), 221-289.

[18] H. Digonnet, L. Silva and T. Coupez, Cimlib: a fully parallel application for numerical simulations based on components assembly, Proceedings of the 9th International Conference on Numerical Methods in Industrial, 2007.

[19] S. Whitaker, The Method of Volume Averaging, Theory and Applications of Transport in Porous Media, Kluwer Academic Publishers, 1999.

[20] K.M. Pillai, Governing equations for unsaturated flow through woven fiber mats. Part 1. Isothermal flows, Compos. Part. A. Appl. S., 33:7 (2002), 1007-1019. 
[21] C.L. Tucker III and Richard B. Dessenberger, Flow and Rheology in Polymer Composites Manufacturing, Governing Equations for Flow and Heat Transfer in Stationary Fiber Beds (Chapter 8), Elsevier Science Publisher, 1992.

[22] K. Hitti, Direct numerical simulation of complex Representative Volume Elements (RVEs): Generation, resolution and homogenization, Ph.D. thesis, Ecole Nationale Supérieure des Mines de Paris, 2011.

[23] M. Bernacki, H. Resk, T. Coupez and R.E. Logé, Finite element model of primary recrystallization in polycrystalline aggregates using a level set framework, Model. Simul. Mater. Sc., 17 (2009), 064006.

[24] E. Hachem, T. Kloczko, H. Digonnet and T. Coupez, Stabilized finite element solution to handle complex heat and fluid flows in industrial furnaces using the immersed volume method, Int. J. Numer. Meth. Fluids, 68 (2012), 99-121.

[25] P. Laure, G. Beaume, O. Basset, L. Silva and T. Coupez, Numerical methods for solid particles in particulate flow simulations, Eur. J. Comp. Mechanics, 16 (2007), 365-383.

[26] L. Franca, A. Nesliturk and M. Stynes, On the stability of residual-free bubbles for convection diffusion problems and their approximation by a two-level finite element method, Comp. Meth. Appl. Mech. Eng., 166 (1998), 35-49.

[27] D. Arnold, F. Brezzi and M. Fortin, A stable finite element for the stokes equations, Calcolo, 21 (1984), 337-344.

[28] K. Hitti, P. Laure, P. Coupez, L. Silva and M. Bernacki, Precise generation of complex statistical Representative Volume Elements (RVEs) in a finite element context, Comp. Mat. Sc., 61 (2012), 224-238.

[29] B. Ripley, Modelling spatial patterns, J. Roy. Stat. Soc. B., 39:2 (1977), 172-212.

[30] B. Ripley, Spatial Statistics, Wiley, New York, 1981.

[31] F. Goreaud and R. Pélissier, On explicit formulas of edge effect correction for ripley's k-function, J. Veg. Sci., 10 (1999), 433-438.

[32] P.J. Diggle, Statistical Analysis of Spatial Point Patterns, 2nd edn, Oxford University Press, Oxford, 2003. 